\title{
Computer Simulation of Vibration Spectrum in Disordered Systems
}

\author{
Z.C. Shen
}

International Centre for Materials Physics, Academia Sinaca, Sheng Yang 110015, Suzhou Professional University, Suzhou, 215002, China

\begin{abstract}
The disordered systems are of fractal structures. Alexander and Orbach showed that the vibrational density of states for fractal nets follows the universal law: $D(\omega) \sim \omega^{\mathrm{d}-\mathrm{i}}$. where $\vec{d}$ is the spectral (fracton) dimensionality; $\bar{d}=4 / 3$ for any Euclidean dimension $d$. The excitations belonging to this class were named "fractons". We have employed a novel numerical method developed by williams et al, and performed computer simulation. We have pointed out that the connection between the vibration spectrum and the ultrasonic attenuation in sintered metal powders.
\end{abstract}

\section{Introduction}

The disordered systems, e. g. .amorphous structures, sintered metal powders and so forth, are important research directions in condensed matter physics. Because the objects of study are not strict periodic structure, the difficulty of theoretical treatment is larger. The concept of fractal structure, developed by Mandelbrot ${ }^{[H]}$, has proven to be of great utility because many structures that appear purely random can be described within a geometric mathematical framework. The development of fractal geometry leads to remarkable advances in the description of many phenomena.

\section{Fracton}

In the present paper, a number of experimental results concerning the acoustical vibrations of self - similar fractal solids are reviewed. We consider fractal solids which are selfsimilar over a range of lengths $l$, comprised between their partical (or moleccular) size a and their correlation length $\xi ; a<1<\xi$. Beyond $\hat{\xi}$, the solids are homogeneous. The long wave acoustical vibrations, of wavelength $\lambda>\xi$, are then acoustical phonons of angular frequency $\omega$, wave vector $k=2 \pi / \lambda$, and density of states $N_{p h}(\omega)=\omega^{d-1}$; where $d$ is the Euclidean dimension of the embedding space. As $\omega$ is continuously increased, one reaches a crossover frequency $\omega_{\text {col }}$, where $K \xi=1$. Near and beyond this point.all length seales relevant to vibrations are expected to collapse to a single one, $\mathrm{Kl}_{\text {loc }} \simeq \mathrm{K} \mathrm{l}_{\text {scar }} \simeq$ $\mathrm{kl} \simeq 1$. These excitations have been called fractons ${ }^{[2]}$, and their density of states was assumed to scale with $\omega$ as $N_{t r}(\omega) \alpha \omega^{d-1}$. Here $\bar{d}$ is the so-called spectral dimension, different from $D$, the Hausdorff dimension of the fractal, $\bar{d}<D$, where $\bar{d}$ is the spectral (fracton) dimensionality, $\bar{d}=4$ / 3 for any Euclidean dimension d. ${ }^{2}$-Fractons are strongly localized eigenmodes.

The understanding of the nature of localized fractons is , however, unsatisfactory both theoretically and experimentlly. Computer simulations were seen to be one of the most convincing experiments revealing the nature of localized fractons.

\section{Method}

We have performed computer experiments at the percolation threshold Pc. Our samples treated have the site-number with $N=1600$, for which a numerical method developed by Williams and Maris $^{[3]}$ was employed.

Let us consider a set of $\mathrm{N}$ atoms which are coupled together by linear springs. For simplicity, we consider the particles to move only in a single direction (e.g. the $z$ direction), but our method 
can easily be extended to cover general motions. The equation of motion of everyone atom is

$$
M_{1} \vec{u}_{1}(t)=-\sum_{1} \varnothing_{11} \cdot u_{1} \cdot(t)
$$

$M_{1}$ and $u_{1}(t)$ are the mass and displacement of 1 th atom and $\Phi_{11}$. describes the strength of the spring coupling atoms 1 and $1^{\prime}$. The displacement can be decomposed into a set of normal modes according to

$$
u_{1}(t)=\sum_{i} Q_{i}(t) \frac{e_{1}(\lambda)}{\sqrt{M_{1}}}
$$

where $Q_{\lambda}$ is the amplitude of the normal mode $\lambda$ and $e_{1}(\lambda)$ is the displacement pattern. We start with each atom at rest and with zero displacement. We then apply a force on each atom given by

$$
F_{1} \cos (\Omega \mathrm{t})
$$

where $F_{1}$ is independent of time. The total energy of the system is

$E=\frac{1}{2} \sum_{\lambda}\left[\sum_{1} \frac{F_{1} e_{1}(\lambda)}{\sqrt{M_{1}}}\right]^{2} \frac{\left.\sin ^{2}\left\{\left[\omega_{\lambda}-\Omega\right) / 2\right] t\right\}}{\left(\omega_{\lambda}-\Omega\right)^{2}}$

Now let us choose

$$
F_{1}=F_{0} \sqrt{M_{1}} \cos \left(\Phi_{1}\right)
$$

where $F_{0}$ is a constant and $\Phi_{1}$ is a random quantity. If we average over all possible values of $\Phi_{\mathrm{i}}$. we find that the average value of the total energy $E$ becomes

$$
<E>=\frac{F_{0}^{2}}{4} \sum \frac{\sin ^{2}\left\{\left[\left(\omega_{\lambda}-\Omega\right) / 2\right] t\right\}}{\left(\omega_{\lambda}-\bar{\Omega}\right)^{2}}
$$

Thus, under certain conditions we can find the density of vibrational states $g(\Omega)$ from

$$
g(\Omega)=\frac{8<E>}{\pi \mathrm{t} F_{0}^{2} \mathrm{~N}}
$$

The problem thus reduces to the solution of equations of motion of the system in he presence of a periodic force. These equations can be written as

$\dot{v}_{1}(\mathrm{t})=\mathrm{M}_{1}^{-1}\left\{-\sum_{1} \varnothing_{11} \mathrm{u}_{\mathrm{1}^{\prime}}(\mathrm{t})+\mathrm{F}_{\mathrm{l}} \cos (\Omega \mathrm{t})\right\}$

$\dot{u}_{3}(t)=v_{1}(t)$

The standard approach to the time development is to replace $t$ by $n \tau$ where $\tau$ is a small time step, and $n$ is an integer. Then a time development algorithm yields

$$
\begin{aligned}
& u_{1}(n+1)=v_{1}(n)+M_{1}^{-1}\left[\sum_{1} \varnothing_{1} u_{1}^{\prime}(n)+F_{1} \cos (\Omega n \tau)\right] \tau \\
& u_{1}(n+1)=u_{1}(n)+v_{1}(n) \tau \\
& \tilde{\omega}=\frac{2}{\tau} \sin ^{-1}\left[\frac{\omega \tau}{2}\right]
\end{aligned}
$$

Thus we can apply this algorithm to the system of $\mathrm{N}$ atoms driven by the force (3). This system can be thought of as $\mathrm{N}$ oscillators, and so the algorithm effectively time develops each of these oscillators with an apparent frequency given by (12).

\section{Results and Discussions}

We have applied our numerical method to study localization in a simple two-dimensional model. The atoms form a simple cubic lattice in the $x-y$ plane, and are allowed to move only in the $Z$ direction. These atoms interact only with nearest neighbors via springs of strength $\mathrm{k}$. If we label the atoms by coordinates $l_{x}, l_{y}$, the equation of motion becomes

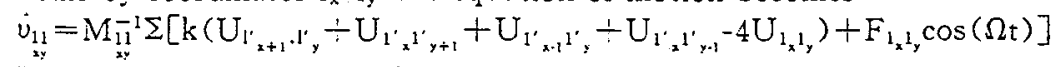

We have calculated $\mathrm{g}(\omega)$ for lattices of 1600 atoms in a $40 \times 40$ array. The spring constant $\mathrm{k}$ was 1 , and the masses were randomly distributed in the range

$1-\mathrm{q}<\mathrm{M}<1+\mathrm{q}$

The computer simulate curve is shown in Fig. 1. It is quite in agreement with the relevant experimental results. ${ }^{[4.5 .6}$. Most noticeable are the experiments on sintered metal powders ${ }^{[4.5 .6]}$. We believe that the very large attenuations obsorved in the previous experiments are associate with a 
transition to localized vibrational modes in sinter, as predicted for a percolating structure at length scales near the percolation correlation length. Effective medium approximation calculations appear to exhibit a large increase in the density of states in the crossover region, the computer simulated curve is consistent with results outlinded above.

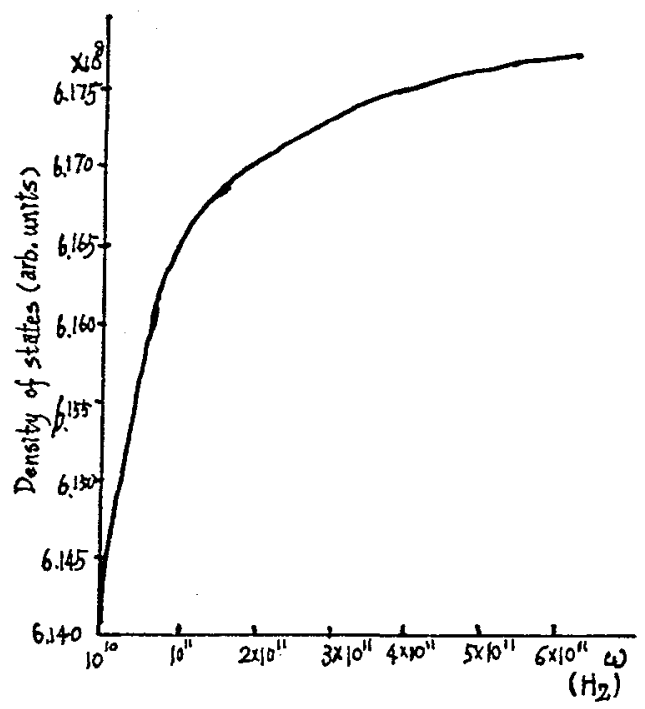

Fig. 1. Computer simulate curve of the density of vibrationai states for $\mathrm{N}=1600$ atoms system

\section{References}

[1]B. B. Mandelbrot, The Fractal Geometry of Nature (Freeman, San Francisco, 1983).

[2]S. Alexander and R. Orbach, J. Phys. (Paris) 43(1982)L625.

[3]M. L. Willians and H. J. Maris. ,Phys. Rev. B 31 (1985) 4508.

[4]E. Courtens, R. Vacher and E. Stoll ,Physica D3s(1989) 41.

[5]D. Deptuck .J. P. Harrison and P.Eawadski, Phys. Rev. Lett. 54(1985) 913.

[6]J. H. Page and R. D. McCullock ,Phys. Rev. Lett. 57(1986)1324. 